\title{
Two-color fluorescent in situ hybridization in the embryonic zebrafish brain using differential detection systems
}

\author{
Gilbert Lauter, Iris Söll and Giselbert Hauptmann*
}

\begin{abstract}
Background: Whole-mount in situ hybridization (WISH) is extensively used to characterize gene expression patterns in developing and adult brain and other tissues. To obtain an idea whether a novel gene might be involved in specification of a distinct brain subdivision, nucleus or neuronal lineage, it is often useful to correlate its expression with that of a known regional or neuronal marker gene. Two-color fluorescent in situ hybridization (FISH) can be used to compare different transcript distributions at cellular resolution. Conventional two-color FISH protocols require two separate rounds of horseradish peroxidase (POD)-based transcript detection, which involves tyramide signal amplification (TSA) and inactivation of the first applied antibody-enzyme conjugate before the second detection round.

Results: We show here that the alkaline phosphatase (AP) substrates Fast Red and Fast Blue can be used for chromogenic as well as fluorescent visualization of transcripts. To achieve high signal intensities we optimized embryo permeabilization properties by hydrogen peroxide treatment and hybridization conditions by application of the viscosity-increasing polymer dextran sulfate. The obtained signal enhancement allowed us to develop a sensitive two-color FISH protocol by combining AP and POD reporter systems. We show that the combination of AP-Fast Blue and POD-TSA-carboxyfluorescein (FAM) detection provides a powerful tool for simultaneous fluorescent visualization of two different transcripts in the zebrafish brain. The application of different detection systems allowed for a one-step antibody detection procedure for visualization of transcripts, which significantly reduced working steps and hands-on time shortening the protocol by one day. Inactivation of the first applied reporter enzyme became unnecessary, so that false-positive detection of co-localization by insufficient inactivation, a problem of conventional two-color FISH, could be eliminated.

Conclusion: Since POD activity is rather quickly quenched by substrate excess, less abundant transcripts can often not be efficiently visualized even when applying TSA. The use of AP-Fast Blue fluorescent detection may provide a helpful alternative for fluorescent transcript visualization, as the AP reaction can proceed for extended times with a high signal-to-noise ratio. Our protocol thus provides a novel alternative for comparison of two different gene expression patterns in the embryonic zebrafish brain at a cellular level. The principles of our method were developed for use in zebrafish but may be easily included in whole-mount FISH protocols of other model organisms.
\end{abstract}

Keywords: digoxigenin, dinitrophenol, tyramide signal amplification (TSA), dextran sulfate, hydrogen peroxide, permeabilization, Fast Red, Fast Blue, alkaline phosphatase, horseradish peroxidase, prosomere

\footnotetext{
* Correspondence: giselbert.hauptmann@ki.se

Karolinska Institutet, Department of Biosciences and Nutrition, SE-141 83

Huddinge, Sweden
} 


\section{Background}

In situ hybridization is the method of choice to characterize the spatial distribution of gene transcripts during embryonic development as well as in adult tissues. Initial protocols used isotope-labeled nucleotide probes for detection of transcripts on tissue sections [1]. A major methodological advance was the introduction of non-radioactive digoxigenin-labeled probes that permitted for the first time to visualize global gene expression patterns in Drosophila embryos [2]. This set the starting point for detection of global transcript distributions in complete tissues, organs and embryos of invertebrate and vertebrate model species. Another milestone was the development of multicolor whole-mount in situ hybridization (WISH) procedures for differential color visualization of two or more mRNAs in one and the same embryo [3-7]. In the original method for zebrafish embryos, digoxigenin- and fluorescein-labeled RNA probes were together hybridized and sequentially visualized by two rounds of alkaline phosphatase (AP) detection using Fast Red and BCIP/NBT as differential colorimetric substrates $[8,9]$. This protocol has been used to compare numerous regulatory gene expression domains in the developing zebrafish brain [10-14].

Fast Red forms a red precipitate, which can be fluorescently visualized using Texas Red or rhodamine filter sets [15]. Fast Red in combination with ELF (enzyme labeled fluorescence) substrate [16] has been used for initial tries of two-color fluorescent in situ hybridization (FISH) based on AP detection in zebrafish and mouse $[17,18]$. However, the low sensitivity and speckled signal of the ELF substrate did not produce satisfactory results, so that a second powerful fluorescent AP substrate for wholemount FISH was missing. Therefore, current wholemount FISH protocols instead apply horseradish peroxidase (POD) and fluorescent tyramide substrates for signal amplification [19]. The signal enhancement in combination with the availability of a number of different fluorescent tyramide substrates made multicolor whole-mount FISH possible [20-25]. However, POD is inactivated by substrate excess, so that enzymatic activity is rather quickly quenched. Consequently, the tyramide signal amplification (TSA) reaction can last productively only for less than 30 minutes. This is often not sufficient for detection of lower expressed transcripts. In contrast, APbased substrate turn over can last over hours because of long-lasting enzymatic activity and high signal-to-noise ratio. Furthermore, because of relatively high background autofluorescence of zebrafish embryos and substrate trapping in the hydrophobic yolk the introduction of the TSA system into multiplex FISH applications for this model organism was problematic. As a consequence, a current protocol tried to compensate for the lowered sensitivity by using additional layers of antibody detection for signal amplification, which made the procedure even longer and more laborious [20].

To make use of the benefits of both, long-lasting enzymatic activity of AP as well as tyramide signal amplification of POD, we aimed to combine the two detection systems for two-color whole-mount FISH. We show here that aside Fast Red also Fast Blue $[4,26]$ produces chromogenic as well as fluorescent signals. To increase signal strength of Fast dyes, we optimized embryo permeabilization properties and hybridization efficiency, respectively, by hydrogen peroxide treatment and addition of dextran sulfate to the hybridization reaction. The resulting increased signal intensities allowed us to combine AP-Fast dye and POD-TSA detection for twochannel fluorescent visualization of different mRNA probes. Because of the different reporter systems applied only one antibody incubation step was necessary and an antibody-enzyme conjugate inactivation step could be omitted resulting in reduced working steps and time spent.

\section{Results and discussion}

\section{Effect of dextran sulfate in AP-based chromogenic WISH}

An essential component of successfully performing a WISH experiment is to achieve reasonably high signal sensitivities. Since addition of viscosity-increasing polymers could improve POD-based FISH signals [25,27], we tested in a pilot experiment whether addition of dextran sulfate to the hybridization reaction would improve signal sensitivity in AP-based WISH. 24-hpf zebrafish embryos were hybridized to a $\operatorname{sim} 1 a$-specific digoxigenin probe with or without addition of $5 \%$ dextran sulfate to the hybridization mix and transcripts were visualized by AP-based BCIP/NBT staining under identical conditions and staining times (Figure 1). Expression of sim1a in the embryonic rostral brain was detected by both samples, but expression sites were much stronger visualized in dextran sulfate treated embryos (Figure 1A,B). Less pronounced expression sites in the basal brain and pronephric primordium could easily be missed in embryos hybridized without dextran sulfate addition (arrowheads in Figure 1A,C) as compared to dextran-treated embryos (Figure 1B,D). The positive effect of dextran sulfate is probably due to a molecular crowding effect that may lead to a local increase of probe concentration [28].

\section{Optimization of Fast dye signal strength}

We previously showed that Fast Red and Fast Blue could be applied to chromogenic visualization of transcripts in WISH experiments in Drosophila and zebrafish embryos $[4,26,29]$. For two-color experiments it is essential to 


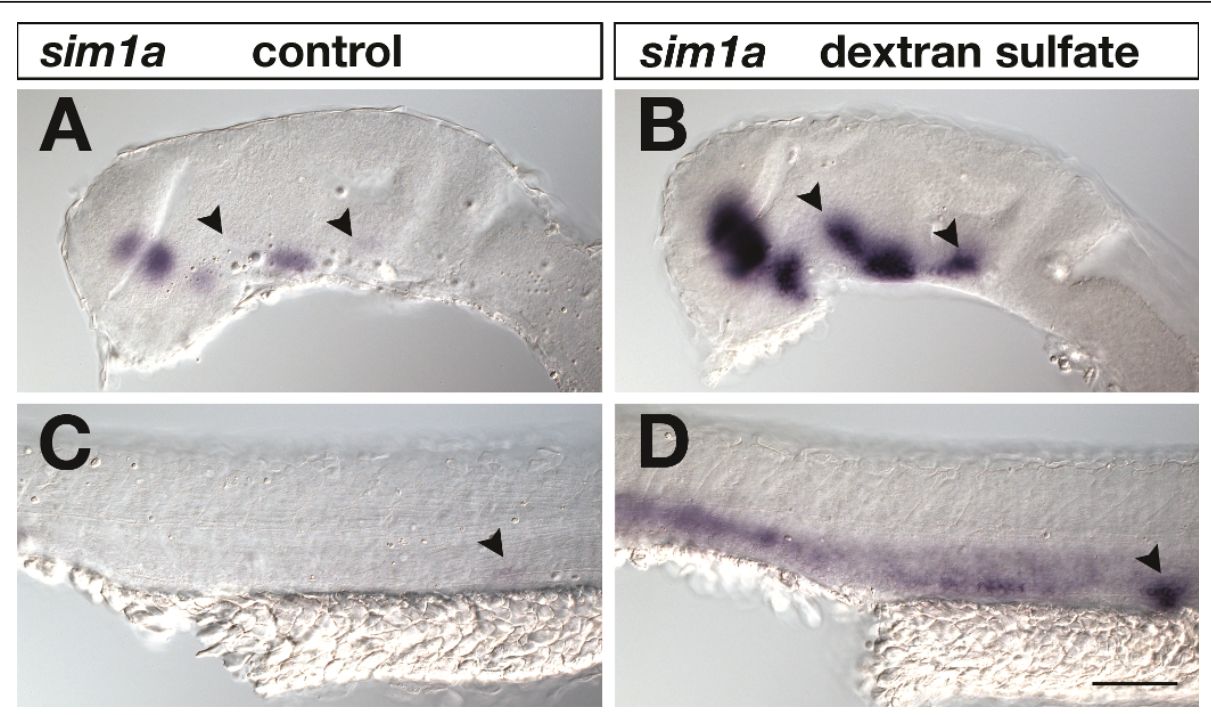

Figure 1 Effects of dextran sulfate in standard WISH. 24-hpf embryos were hybridized to a sim 1a digoxigenin-labeled RNA probe, which was visualized by AP-BCIP/NBT chromogenic detection under identical conditions and staining times. Lateral views of embryonic brains (A,B) and trunks $(C, D)$ are shown with anterior to the left. In $(B, D)$ but not in $(A, C) 5 \%$ dextran sulfate was included in the hybridization buffer. Addition of dextran sulfate resulted in increased signal sensitivity. Arrowheads in $(A, B)$ indicate simla-positive neuronal clusters identified in dextran sulfate treated brains (B) that were hardly detected in untreated embryos (A). Arrowheads in (C,D) mark the pronephric primordium strongly visualized in dextrane sulfate treated embryos (D) but not in untreated specimens (C). Embryos were viewed on an Axioplan II microscope and images were recorded with an Axiocam digital camera. Scale bar is $100 \mu \mathrm{m}$.

achieve high signal intensities and signal-to-noise ratios with both probes. However, Fast dyes are less sensitive than standard BCIP/NBT staining. We therefore tested whether presence of dextran sulfate in the hybridization mix would improve signal intensity when using Fast Red or Fast Blue as substrates. 24-hpf zebrafish embryos were hybridized to a dinitrophenol-labeled antisense RNA probe specific for shha and visualized using Fast Red or Fast Blue as substrates (Figure 2). Both, Fast Red and Fast Blue substrate deposition was dramatically increased in dextran sulfate treated (Figure 2C,G) as compared to untreated embryos (Figure 2A,E) under otherwise identical conditions and staining times.

To further improve signal sensitivities we aimed to improve accessibility of embryos for probes and antibody-enzyme conjugates. In previous experiments we made use of hydrogen peroxide to block endogenous peroxidase activity of embryos. We thereby noticed that this treatment lead to improved signal intensities. Because hydrogen peroxide can disrupt cell membranes, we sought to explore the possibility whether hydrogen peroxide would be useful to improve embryo permeabilization properties. Prior to standard proteinase $\mathrm{K}$ digestion, we treated the fixed embryos with $2 \%$ hydrogen peroxide. Hydrogen peroxide treatment resulted in slightly improved signal detection (Figure 2B,F) and when hydrogen peroxide permeabilized embryos were hybridized in the presence of dextran sulfate strongest signal intensities were obtained (Figure 2D,H).
Next, we applied Fast Blue in combination with Fast Red to chromogenic two-color detection of $n k x 6.1$ and pax6a (Figure 3E). Expression of $n k x 6.1$ was visualized by AP-Fast Blue staining. After inactivation of the first applied antibody-AP conjugate, pax6a expression was revealed by AP-Fast Red chromogenic reaction. In APbased two-color experiments the second detection round is less sensitive than the first one, so that the positive effects of hydrogen peroxide for improved embryo permeabilization were even more obvious. Chromogenic detection of pax6a transcripts by AP-Fast Red as second round staining revealed that an optimal signal was obtained within 4 hours staining time in hydrogen peroxide permeabilized embryos. In contrast, embryos that were not permeabilized with hydrogen peroxide developed barely detectable chromogenic signals within the same staining time. Comparably strong signals were however obtained after prolonged Fast Red staining of 12 hours (not shown).

\section{Chromogenic and fluorescent WISH using Fast dyes as AP substrates}

Fast Red forms a red precipitate that can be fluorescently visualized using rhodamine filter sets as exemplified here by chromogenic and fluorescent detection of pax6a transcript distribution in the forebrain and hindbrain at $24 \mathrm{hpf}$ (Figure 3A,B). To test whether Fast Blue is comparably useful for fluorescent mRNA detection, we hybridized 24-hpf zebrafish embryos with an $n k x 6.1$ - 

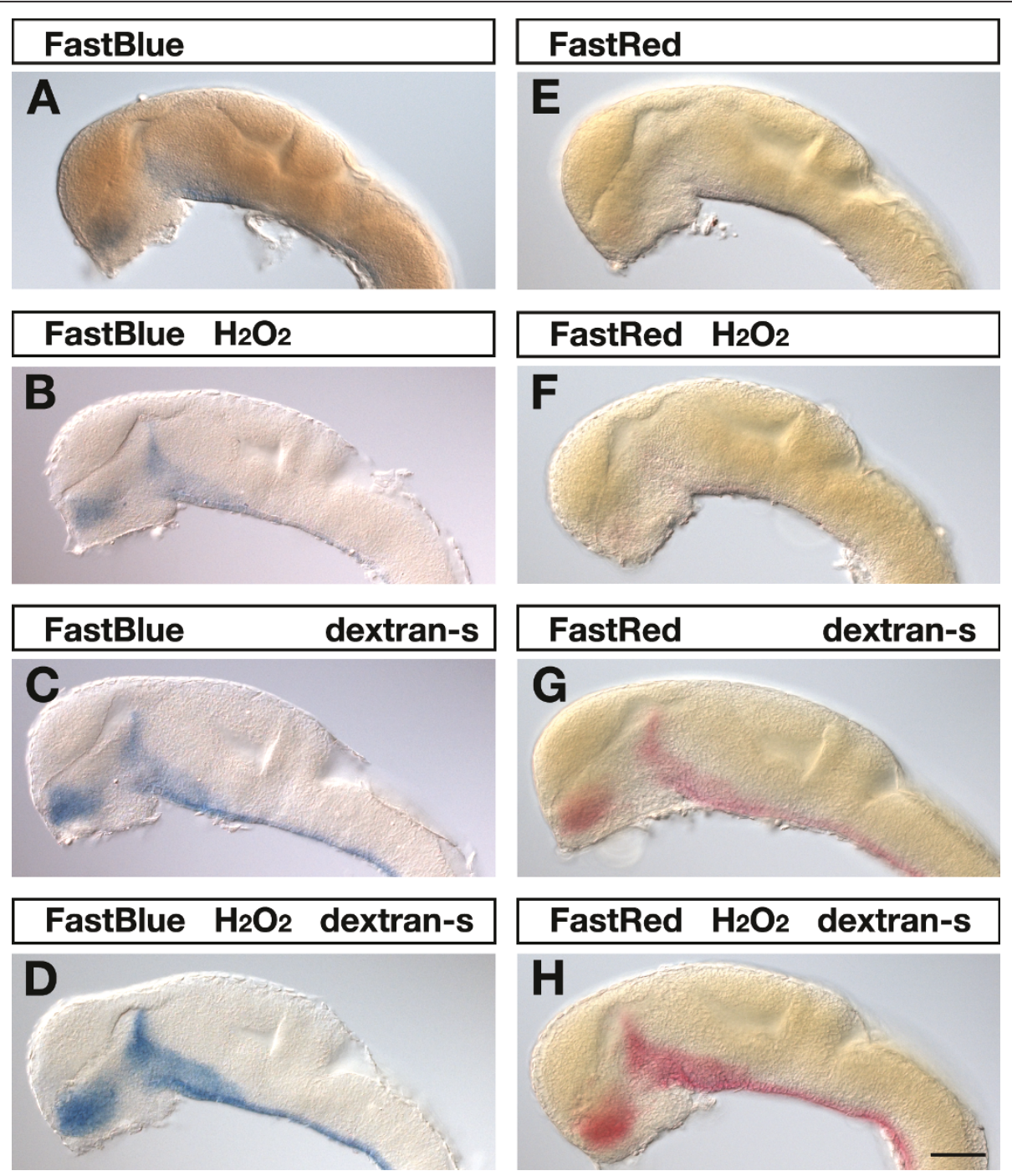

Figure 2 Effects of hydrogen peroxide and dextran sulfate on Fast Red and Fast Blue detection. 24-hpf embryos were hybridized to a shha dinitrophenyl-labeled RNA probe, which was visualized by AP-Fast Blue (A-D) or AP-Fast Red (E-H) chromogenic staining for 55 min and 80 min, respectively. Lateral views of embryonic brains are shown with anterior to the left. On top of each panel it is indicated whether embryos were permeabilized with $2 \%$ hydrogen peroxide $\left(\mathrm{H}_{2} \mathrm{O}_{2}\right)$ and whether $5 \%$ dextran sulfate (dextran-s) was added to the hybridization buffer Strongest signals were obtained when hydrogen peroxide treated embryos were hybridized in the presence of dextran sulfate $(D, H)$. Embryos were viewed on an Axioplan II microscope and images were recorded with an Axiocam digital camera. Scale bar is $100 \mu \mathrm{m}$.

specific digoxigenin probe followed by AP-Fast Blue detection (Figure 3C,D). Expression of $n k x 6.1$ in the basal fore- and midbrain and along the ventral hindbrain and spinal cord could be clearly shown by Fast Blue precipitate deposition (Figure 3C) as well as fluorescent visualization using far-red filter sets (Figure 3D). Fluorescent detection (Figure 3D) revealed corresponding transcript distributions as those obtained with chromogenic substrate deposition (Figure 3C) confirming the specificity of the fluorescent signal.

It is certainly possible to combine Fast Blue and Fast Red for chromogenic two-color WISH $[4,26,29]$ and we revealed clearly abutting expression patterns of $n k x 6.1$ and pax6a in the experiment presented (Figure 3E). However, two-color fluorescent visualization of transcripts cannot be recommended using both Fast dyes together, since Fast Red and Fast Blue display overlapping red fluorescence emission, leading to significant bleed-through between channels (data not shown). In addition, the two chromogenic precipitates formed during the AP-reaction can obscure each other's fluorescent signal. Therefore, it may be more advantageous to combine AP-Fast dye and POD-TSA fluorescent detection systems. 

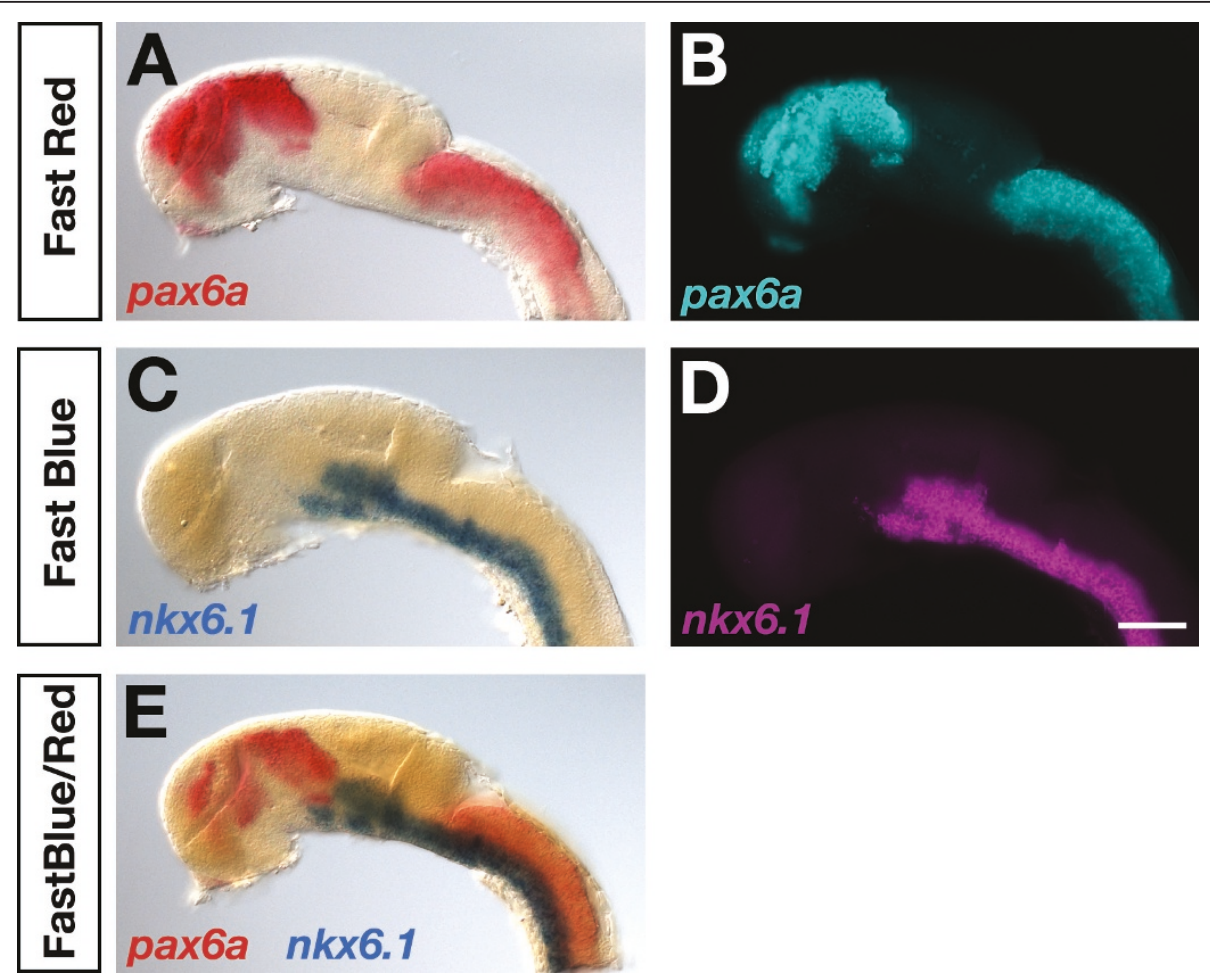

Figure 3 Fast Red and Fast Blue permit chromogenic and fluorescent transcript visualization. Lateral views of embryonic brains at 24 hpf hybridized to $n k \times 6.1$ digoxigenin and/or pax6a dinitrophenol antisense RNA probes as indicated on each panel. Transcript distributions were visualized by chromogenic $(A, C, E)$ or fluorescent $(B, D)$ detection of Fast Blue $(C, D, E)$ and Fast Red $(A, B, E)$ precipitates as indicated. Embryos were viewed on an Axioplan II microscope and images were recorded with an Axiocam digital camera. Fluorescent signals were false-colored in ImageJ. Scale bar is $100 \mu \mathrm{m}$.

\section{Combination of AP and POD systems}

We tested the versatility of combining AP and POD substrate reactions for fluorescent detection of different transcripts. A prerequisite of using Fast dyes in twocolor FISH is to identify whether there is bleed-through [30] between the chosen detection windows. Since zebrafish embryos have significantly high cyan and UV, but rather low red and green autofluorescence [20], we sought to best combine Fast dye and green TSA-carboxyfluorescein (FAM) for two-channel fluorescence detection.

In single-color FISH experiments, pax6a and $n k x 6.1$ were visualized by Fast Red and TSA-FAM fluorescence detection, respectively, and each expression pattern was recorded in both detection channels (Channel01: wavelengths greater than $560 \mathrm{~nm}$ were collected; Channel02: wavelengths between $505 \mathrm{~nm}$ and $545 \mathrm{~nm}$ were collected). Forebrain expression of pax6a was appropriately visualized in the Fast Red (Ch01; Figure 4A) and not in the TSA-FAM detection channel (Ch02; Figure 4B). In contrast, $n k x 6.1$ expression in the fore- and midbrain was visualized in both, the TSA-FAM (Figure 4D) and the Fast Red (Figure 4C) detection channel, although somewhat weaker in the latter one. This indicated significant bleed-through of TSA-FAM fluorescence into the Fast Red channel. These results strongly suggested that caution had to be taken to exclude false-positive co-localization, when choosing these two substrates in a two-color FISH experiment.

There was no bleed-through observed, however, between the Fast Blue and TSA-FAM detection channels (Channel01: wavelengths greater than $650 \mathrm{~nm}$ were collected; Channel02: wavelengths between $505 \mathrm{~nm}$ and $545 \mathrm{~nm}$ were collected). Detection of pax6a by Fast Blue revealed a specific signal in the appropriate (Ch01; Figure $5 \mathrm{~A}$ ) but not in the TSA-FAM detection channel (Ch02; Figure 5B). Despite a very strong $n k x 6.1$ TSAFAM signal was visualized (Ch02; Figure 5D), it could not be detected in the Fast Blue detection channel (Ch01; Figure 5C). These results demonstrated the versatility of combining Fast Blue and TSA-FAM for two-color FISH.

\section{Application of Fast dyes in two-color FISH}

We combined POD-TSA-FAM and AP-Fast Red (Figure 6A-C) or AP-Fast Blue (Figure 6D-F) detection systems to directly compare expression of $n k x 6.1$ and pax6a in the same embryo. As described previously $[10,11]$, 


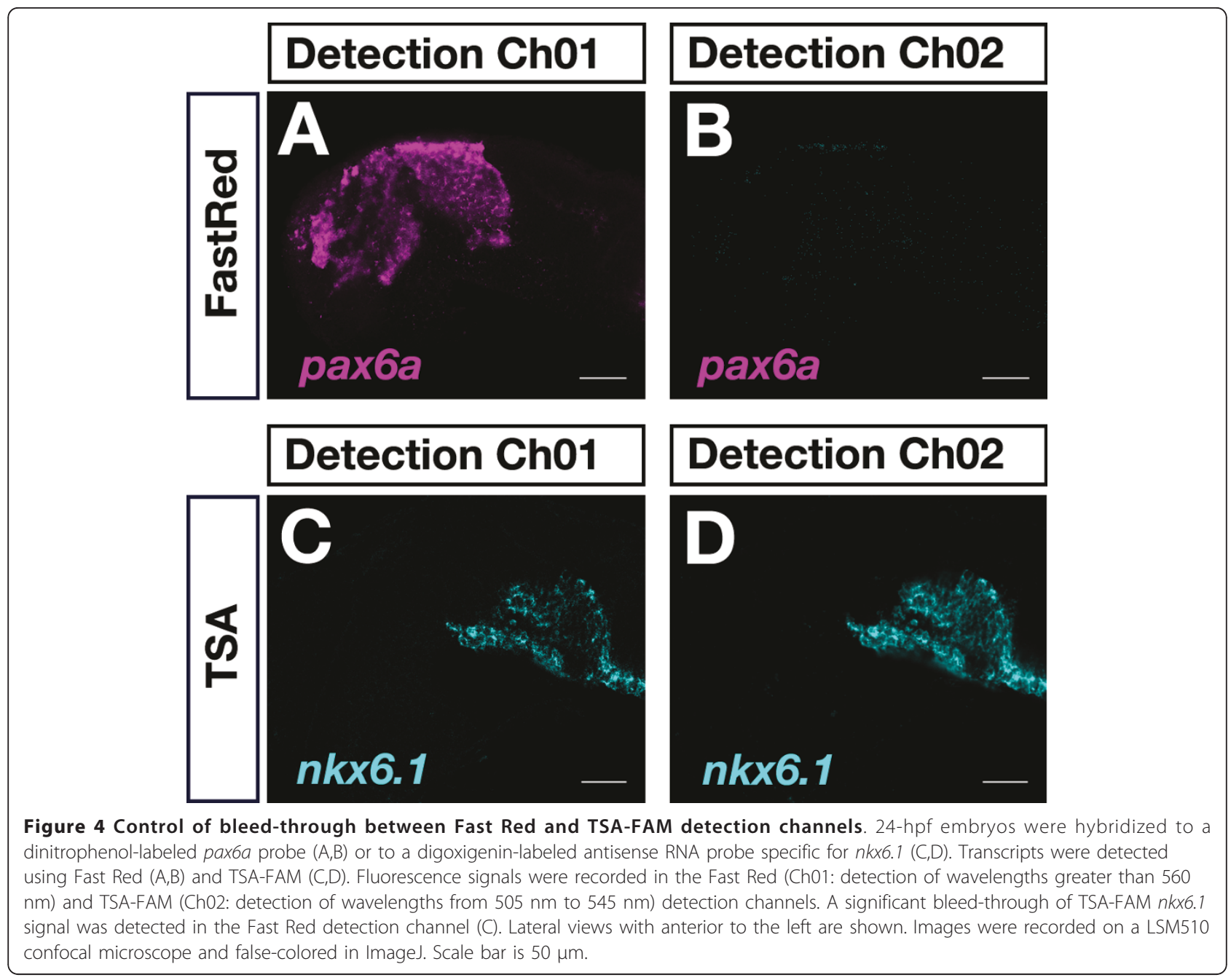

expression of pax6a was confined to distinct domains in the telencephalon and diencephalon. In the alar diencephalon, expression of pax6a was confined to the prethalamus/prethalamic eminence, thalamus/epithalamus and pretectum (Figure 6A,D) corresponding to alar parts of prosomeres 3,2 , and 1 ( $\mathrm{p} 3, \mathrm{p} 2$ and $\mathrm{p} 1$ ), respectively [31,32]. In addition, a separate pax6a cell group was found in the mantle layer of the pretectal basal plate. Expression of $n k x 6.1$ extended in a longitudinal domain along the ventral brain (Figure 6B,E). From the midbrain tegmentum $n k x 6.1$ expression extended rostrally into the diencephalic basal plate beneath the pretectal pax6a expression domain (Figure 6C,F). Interestingly, $n k x 6.1$ expression precisely spared out the basal pax6a stripe confined to b1 (basal p1) and extended in a more ventral domain up to the b1/b2 boundary. In addition, few $n k x 6.1$-positive cells were located more rostrally beneath the pax6a-positive thalamus and anterior to the basal pax6a domain (Figure 6C,F). These $n k x 6.1$ cells were thus localized to b2 (basal p2). Our results indicate that $n k x 6.1$ expression is consistent with prosomeric subdivisions of the basal zebrafish diencephalon [33]. Furthermore, basal forebrain expression of zebrafish $n k x 6.1$ is very similar to that of orthologous genes. In amniotes, Nkx6.1 expression was reported to extend anteriorly into the region of basal $\mathrm{p} 1$ and $\mathrm{p} 2$ [34]. This suggests a conservation of $N k x 6.1$ gene expression in the basal diencephalon of anamniotes and amniotes. In conclusion, our results demonstrate the versatility of using Fast dye fluorescent detection in combination with POD-TSA-FAM in two-color FISH in order to compare differently localized gene expression domains at high resolution (step-by-step protocol, Additional file 1).

When comparing Fast Red and Fast Blue fluorescence signals we noted a sharp localization of the Fast Blue signal that allowed cellular resolution of transcript visualization (Figure 5A, 6D) while Fast Red sometimes produced a little less localized signal (Figure 4A, 6A). This suggested that Fast Red was more prone to diffusion of reaction products away from the site of enzymatic activity 


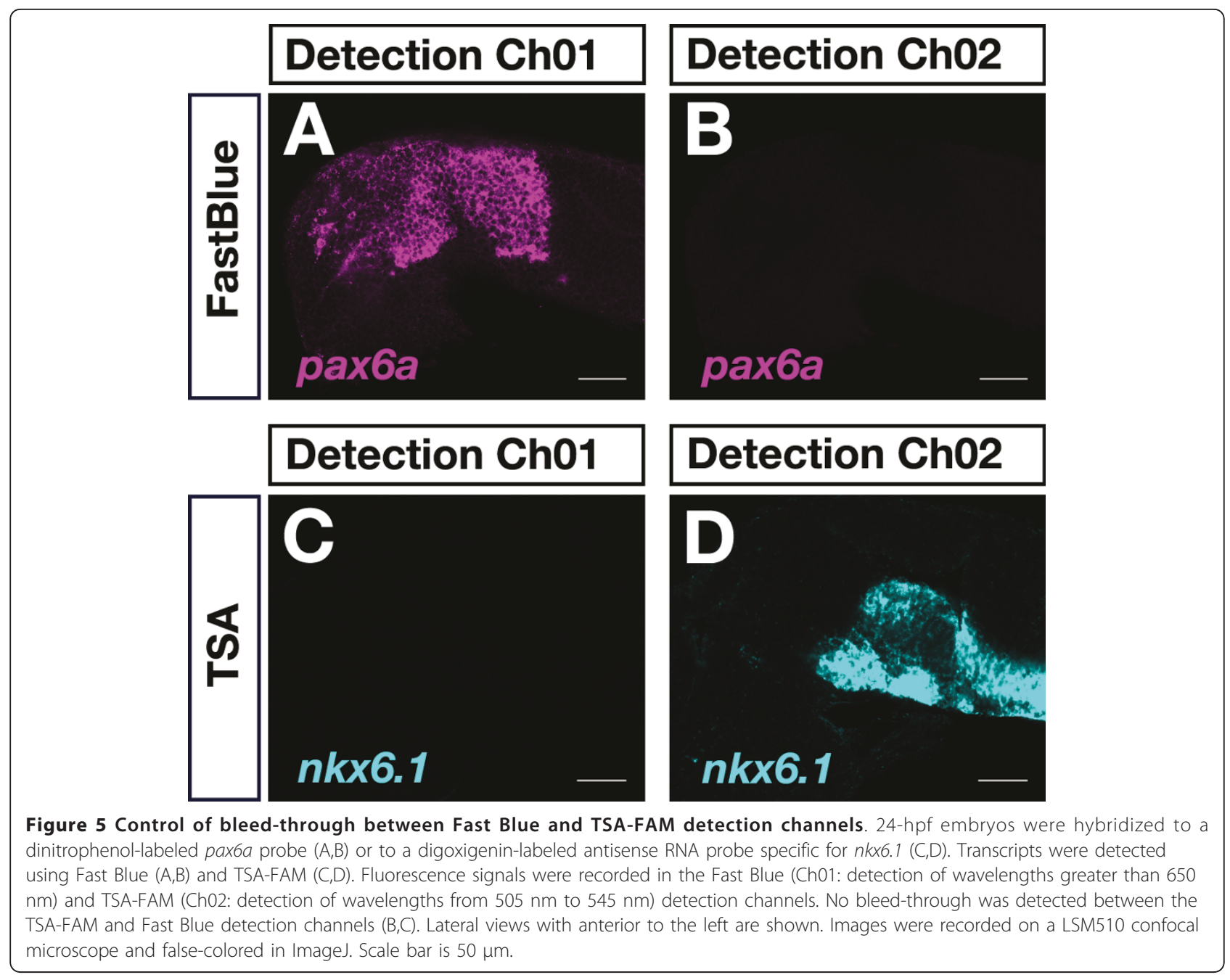

before precipitation, which may affect target resolution. In addition, prolonged Fast Red staining times could result in formation of spurious crystals (Figure 4A) and nonspecific background staining, while this was less of a problem with Fast Blue. In our hands, Fast Blue seemed slightly more sensitive than Fast Red as we obtained comparable signals within shorter staining times (55 versus 80 minutes for embryos in Figure 2D,H).

We showed that AP-based Fast Blue fluorescent detection in the far-red can be optimally combined with POD-based green fluorogenic TSA substrates excluding crosstalk and bleed-through in two-color experiments. Since Fast Blue emission is in the far-red, it can easily be combined with green and blue fluorochromes in two-channel fluorescence detection experiments. Fast Red, which has an emission spectrum closer to the green FAM resulting in potential bleed-through (Figure 4C), may be combined with cyan fluorochromes (e.g. Alexa Fluor 410). However, there is high UV and significant cyan autofluorescence in zebrafish embryos, which makes fluorescent signals in these channels more difficult to be resolved from background [20].

Current multiplex FISH methods [21-25] require serial rounds of enzymatic amplification and effective inactivation, potentially leading to progressive degradation of samples and reduced sensitivities of subsequent detection rounds. These difficulties may be circumvented by application of different enzymatic detection systems, as described here, allowing for one-step antibody detection. Another recent multiplexed FISH method used in situ hybridization chain reaction (HCR) for signal amplification [35]. HCR amplifiers operate independently in the same sample at the same time, allowing simultaneous detection of multiple target mRNAs, so that sample degradation of sequential detection procedures can be elegantly avoided. Another feature of this method is the use of short RNA probes and hairpins that are supposed to easily penetrate sample tissues. In this study, we optimized embryo permeabilization properties by hydrogen peroxide treatment and hybridization conditions by 

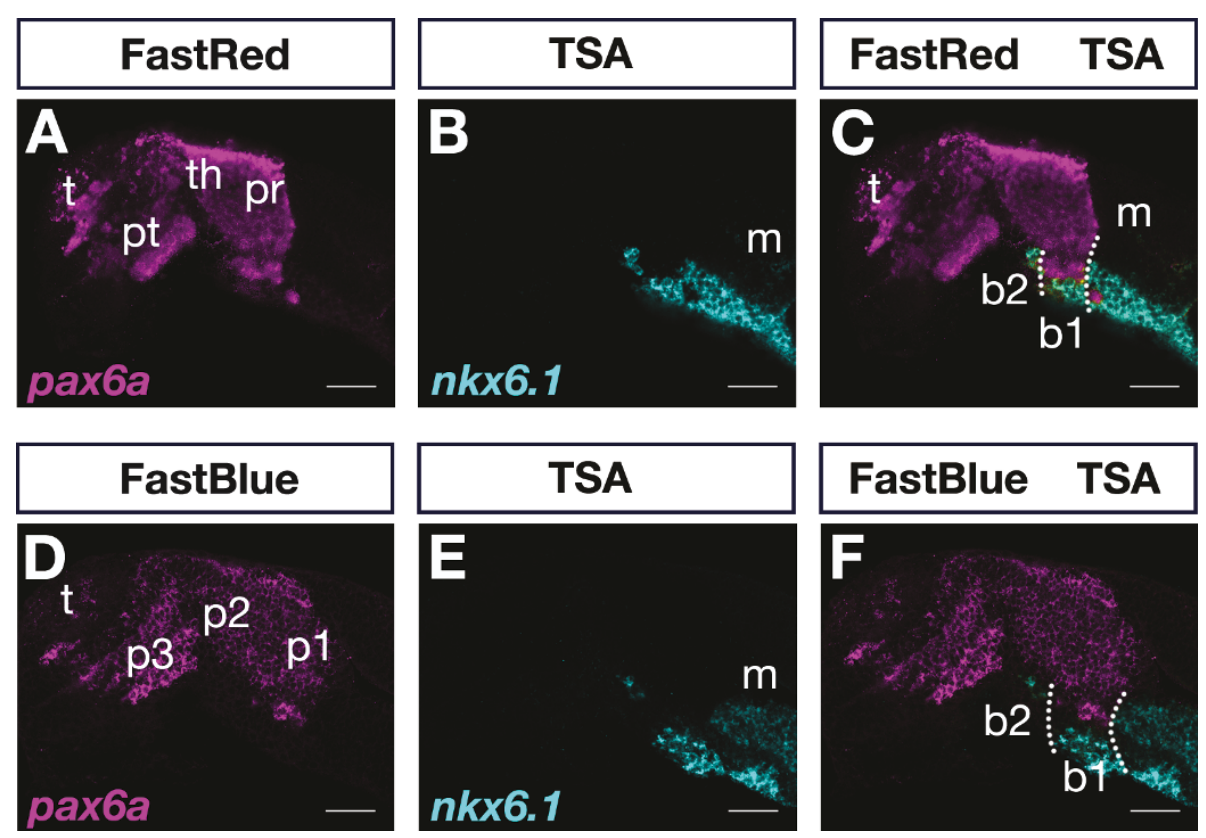

Figure 6 Two-color FISH by combining POD and AP detection systems. 24-hpf embryos were simultaneously hybridized to differentlylabeled pax6a and $n k x 6.1$ probes and visualized by sequential recording of TSA-FAM $(B, C, E, F)$ and Fast Red $(A, C)$ or Fast Blue $(D, F)$. Single channels $(A, B, D, E)$ and overlays of two channels $(C, F)$ are shown. Detection channels for Fast Blue, Fast Red and TSA-FAM were $>650 \mathrm{~nm},>560$ $\mathrm{nm}$, and $505 \mathrm{~nm}$ to $545 \mathrm{~nm}$, respectively. Dotted lines indicate boundaries of basal part of prosomere 1. Abbreviations: b1, basal part of prosomere 1; b2, basal part of prosomere 2; $\mathrm{m}$, midbrain; $\mathrm{p} 1$, prosomere 1; p2, prosomere 2, p3 prosomere 3; pr, pretectum; pt, prethalamus; t, telencephalon; th, thalamus. Images were recorded on a LSM510 confocal microscope and false-colored in ImageJ. Scale bar is $50 \mu \mathrm{m}$.

application of the viscosity-increasing polymer dextran sulfate for increased signal intensities. These measurements may be similarly beneficial for sample penetration and hybridization of short RNA probes and hairpins as described by [35] as well as locked nucleic acid (LNA)modified DNA probes used for detection of miRNAs [36].

\section{Conclusions}

One advantage of our protocol is that POD and AP coupled antibodies can be simultaneously applied, so that just one detection step is required and inactivation of antibody-reporter enzyme conjugates is omitted. Therefore, hands-on time and duration of the complete procedure are considerably reduced and the problem of false-positive signals by insufficient reporter enzyme inactivation is eliminated. When dextran sulfate was included in the hybridization mixture and hydrogen peroxide permeabilized embryos were used the staining time to achieve a strong signal was significantly shortened, which was advantageous for minimizing upcoming background and improving signal-to-noise ratio.

Since POD activity is rather quickly quenched by excess substrate, less abundant transcripts can often not be efficiently visualized despite tyramide signal amplification [19]. AP-Fast Blue fluorescent detection may provide a helpful alternative, as no enzyme inactivation is associated with the AP substrate reaction. Therefore, signal development can be extended at a high signal-tonoise ratio resulting in stronger signal intensities. The applicability of Fast Blue in fluorescent RNA visualization increases the number of possible substrate combinations to choose from. This may allow improved adjustment of detection systems and substrate combinations according to individual requirements of a specific two-color FISH experiment (Table 1). In addition, Fast Red and Fast Blue permit colorimetric as well as fluorescent transcript visualization, so that both types of data can be photographically documented and compared. This can be of advantage as fluorescence detection alone lacks histological context. Thus, our protocol provides a useful alternative method to study expression patterns in relation to each other. We applied our method for comparison of expression domains of neural specific regulatory genes, but this protocol will also be useful for two-color detection of transcripts in other tissues or organs and may be adapted for use in various model organism aside zebrafish.

\section{Methods}

Fish housing

Zebrafish were kept at $26^{\circ} \mathrm{C}$ in an aquaria facility from Schwarz Aquarienbau (Göttingen, Germany) under a 10 
Table 1 Application of different detection systems in two-color WISH

\begin{tabular}{|c|c|c|}
\hline $\begin{array}{l}\text { Detection } \\
\text { System } 1 \\
\end{array}$ & $\begin{array}{l}\text { Detection } \\
\text { System } 2 \\
\end{array}$ & Applications \\
\hline $\begin{array}{l}\text { POD-TSA- } \\
\text { DyLight633 }\end{array}$ & POD-TSA-FAM & $\begin{array}{l}\text { - Routinely used for fluorescent co-localization analysis of two mRNAs at cellular resolution } \\
\text { - If one of the two probes cannot be detected by POD-TSA, combine POD and AP detection }\end{array}$ \\
\hline POD-TSA-FAM & AP-Fast Red & $\begin{array}{l}\text { - If one probe cannot be visualized by POD-TSA this probe may be detected by AP-Fast Red allowing for long } \\
\text { staining times with high signal-to-noise-ratios } \\
\text { - Combine fluorescence and DIC optics, if histological context is required } \\
\text { - Fast Red is preferably used for the more locally distributed mRNA }\end{array}$ \\
\hline POD-TSA-FAM & AP-Fast Blue & $\begin{array}{l}\text { - If there is significant bleed-through between TSA-FAM and AP-Fast Red, AP-Fast Blue as second detection } \\
\text { system may be applied instead } \\
\text { - If the weaker probe cannot be detected by TSA-FAM or Fast Red, prolonged Fast Blue staining may be helpfu } \\
\text { for visualization } \\
\text { - Combine fluorescence and DIC optics, if histological context is required } \\
\text { - Fast Blue is preferably used for the more locally distributed mRNA }\end{array}$ \\
\hline AP-Fast Blue & AP-Fast Red & $\begin{array}{l}\text { - For fluorescent visualization of non-overlapping expression domains } \\
\text { - For colorimetric detection of two mRNAs } \\
\text { - Permits long term storage of stained embryos }\end{array}$ \\
\hline AP-Fast Red & AP-BCIP/NBT & $\begin{array}{l}\text { - Routinely used for colorimetric co-distribution analysis of two mRNAs } \\
\text { - Overlap of two mRNA distributions may be difficult to visualize at cellular resolution (for this purpose } \\
\text { sectioning may be required) } \\
\text { - Combination of fluorescent and chromogenic detection is possible } \\
\text { - Permits long-term storage of stained embryos }\end{array}$ \\
\hline
\end{tabular}

hour dark 14 hour light cycle. Embryos were obtained through natural mating in a 1 liter breeding trap, raised at $28.5^{\circ} \mathrm{C}$ and staged in hours-post-fertilization (hpf). Experiments were performed using only fixed specimen. All experiments were in accordance with ethical permits by Stockholms södra djurförsöksetiska nämnd (Stockholm south ethical council) and jordbruksverket (Swedish board of agriculture).

\section{RNA probes}

Zebrafish sim1a [37], shha [38], pax6a [39] and $n k x 6.1$ [40] cDNA plasmids were kindly provided by Fabrizio Serluca, Denis Duboule, Andreas Püschel and Judith Eisen, respectively. Digoxigenin and dinitrophenollabeled [41] antisense mRNA probes were generated by T7/T3 in vitro transcription [5]. In order to avoid background staining unincorporated nucleotides were removed from the probe preparation. We routinely used column purification of RNA probes [8] according to the manufacturer's instructions (Omega Bio-Tek: Norcross, GA, USA R6249-02).

\section{Embryo pretreatment and hybridization}

Embryos were fixed in $4 \%$ paraformaldehyde at $4^{\circ} \mathrm{C}$ overnight and stored in methanol at $-20^{\circ} \mathrm{C}$. 24-hpf embryos were permeabilized in $2 \%$ hydrogen peroxide in methanol for 20 minutes ( $\mathrm{min}$ ) at room temperature (RT) and stepwise rehydrated to PBST (phosphate buffered saline, $0.1 \%$ Tween-20 pH 7.3). After rehydration, embryos were further permeabilized by a 10 -min proteinase $\mathrm{K}$ treatment and postfixed for 20 min with $4 \%$ paraformaldehyde at RT. Prehybridization, probe hybridization and washes were performed as described [5] except for addition of 5\% dextran sulfate to the hybridization buffer. In two-color experiments, digoxigenin- and dinitrophenol-labeled probes were mixed together in hybridization buffer at the appropriate concentrations and hybridized simultaneously.

\section{Sequential alkaline phosphatase detection using Fast dyes}

Hybridized embryos were blocked with $8 \%$ sheep serum (Sigma: St. Louis, MO, USA S2263) in PBST for at least 1 hour at RT. The digoxigenin probe was detected by incubation for 2 or more hours at RT (or at $4^{\circ} \mathrm{C}$ overnight) with anti-digoxigenin $\mathrm{F}_{\mathrm{AB}}$ fragments (Roche Scandinavia: Bromma, Sweden 11093274910) conjugated to AP diluted 1:4500. Transcripts were visualized in $1 \mathrm{mg} /$ $\mathrm{ml}$ Fast Red TR and $0.4 \mathrm{mg} / \mathrm{ml}$ naphtol-AS-MX-phosphate (NAMP) in $0.1 \mathrm{M}$ Tris- $\mathrm{HCl} \mathrm{pH} \mathrm{8.2.} \mathrm{Before} \mathrm{the} \mathrm{sec-}$ ond detection round, the antibody-AP conjugate was inactivated by $0.1 \mathrm{M}$ glycine- $\mathrm{HCl} \mathrm{pH} 2.2$ treatment for 10 min at RT [8]. The dinitrophenol-labeled probe was detected by incubation for 2 or more hours at RT (or at $4^{\circ} \mathrm{C}$ overnight) with anti-dinitrophenol antibodies coupled to AP (Vector laboratories: Burlingame, CA, USA MB$3100)$ diluted 1:1000. The second mRNA probe was visualized in $0.25 \mathrm{mg} / \mathrm{ml}$ Fast Blue BB (Sigma F3378) and $0.25 \mathrm{mg} / \mathrm{ml}$ NAMP (Sigma N5000) in SB8.2 (0.1 M Tris$\mathrm{HCl} \mathrm{pH} \mathrm{8.2,} \mathrm{containing} 50 \mathrm{mM} \mathrm{MgCl}, 100 \mathrm{mM} \mathrm{NaCl}$, $0.1 \%$ Tween-20) $[4,26]$. Orders of probe label, antibody detection and substrates can be exchanged according to individual requirements.

\section{Combination of AP and POD detection}

Hybridized embryos were blocked with $8 \%$ sheep serum in PBST for at least 1 hour at RT. The digoxigenin and 
dinitrophenol labeled probes were simultaneously detected by incubation for 2 or more hours at RT (or at $4^{\circ} \mathrm{C}$ overnight) in a mixture of anti-digoxigenin POD fragments (Roche 11207733910) diluted 1:500 and antidinitrophenol AP conjugates 1:1000 (Vector laboratories MB-3100). Alternatively, embryos were incubated in a mixture of anti-digoxigenin $\mathrm{AP} \mathrm{F}_{\mathrm{AB}}$ fragments (Roche 11093274910) diluted 1:4500 and anti-dinitrophenol POD (Perkin Elmer: Waltham, MA, USA TSA Plus DNP System NEL747A001KT) conjugates diluted 1:100. For visualization of the two different transcript probes, the PODTSA reaction was performed first followed by AP-Fast Red or AP-Fast Blue staining. Stained embryos were washed by short rinses in TNT $(100 \mathrm{mM}$ Tris- $\mathrm{HCl} \mathrm{pH}$ 7.5, $150 \mathrm{mM}$ Sodium chloride, $0.1 \%$ Tween-20) and PBST.

\section{TSA reaction}

Embryos were washed two times in $100 \mathrm{mM}$ borate $\mathrm{pH}$ 8.5, $0.1 \%$ Tween-20 and incubated in freshly prepared TSA reaction buffer $(100 \mathrm{mM}$ borate $\mathrm{pH} 8.5,2 \%$ dextran sulfate, $0.1 \%$ Tween-20, $0.003 \% \mathrm{H}_{2} \mathrm{O}_{2}$ ) containing bench-made FAM tyramide reagent at a 1:250 dilution $[25,42]$. The TSA reaction was allowed to run for maximal 30 min protected from light and without agitation. Samples were rinsed thoroughly four times in PBST. For each washing step the tubes were inverted several times.

\section{AP substrates}

BCIP 5-bromo-4-chloro-3-indolylphosphate (AppliChem: Darmstadt, Germany A1117), NBT nitro-bluetetrazolium chloride (AppliChem A1243), and Fast Blue BB 4-benzoylamino-2,5-diethoxybenzenediazonium chloride hemi [zinc chloride] salt (Sigma F3378) were dissolved in dimethyl formamide and kept as 50 or $100 \mathrm{mg} / \mathrm{ml}$ stock solutions at $-20^{\circ} \mathrm{C}$. NAMP 3-hydroxy-2-naphthoic acid 2,4-dimethylanilide phosphate (Sigma N5000) was kept as a 50 or $100 \mathrm{mg} / \mathrm{ml}$ stock in dimethyl sulfoxide at $-20^{\circ}$ C. Staining solutions were prepared just prior to use. Two separate solutions of $500 \mu \mathrm{g} / \mathrm{ml}$ Fast Blue and 500 $\mu \mathrm{g} / \mathrm{ml}$ NAMP in SB8.2 were prepared first. The two double-concentrated solutions were then carefully mixed to achieve the final working concentration of $250 \mu \mathrm{g} / \mathrm{ml}$ per substrate. Fast Red/NAMP was obtained in tablet form (Sigma tablet set F4648) and prepared according to the manufacturer's instructions. The Fast Red working solution was filtered to remove non-dissolved substrate particles.

\section{Microscopic visualization}

Embryos were mounted in $75 \%$ glycerol and viewed under a Axioplan2 microscope (Carl Zeiss: Jena, Germany). Photographs were taken with an Axiocam color digital camera (Figure 1, 2 and 3). For fluorescence detection of
Fast Red and Fast Blue, rhodamine and far-red filters were used, respectively. For high-resolution confocal microscopy embryos were mounted in $75 \%$ glycerol in TNT pH 8 containing 1\% low-melting agarose (Figure 4, 5 and 6). Fluorescent signals were recorded on a Carl Zeiss LSM510 confocal microscope using the $633 \mathrm{~nm}$ laser lines for Fast Blue, $543 \mathrm{~nm}$ for Fast Red, and $488 \mathrm{~nm}$ for TSA-FAM for excitation. Fast Blue, Fast Red and TSA-FAM fluorescence emissions were detected using high pass filters LP505, LP560 and LP650 that pass wavelengths greater than 505 $\mathrm{nm}, 560 \mathrm{~nm}$ and $650 \mathrm{~nm}$ to the detector, respectively. Images were processed in opensource Image software to adjust outliers and intensity levels before running a Gaussian smoothening filter. Black-and white pictures were false-colored with the help of ImageJ software. Figure panels were assembled using Adobe Photoshop software.

\section{Additional material}

Additional file 1: Two-color FISH protocol combining POD and AP detection systems. This file contains a step-by-step protocol for use in the laboratory.

\section{Abbreviations}

AP: alkaline phosphatase; FAM: carboxyfluorescein; FISH: fluorescent in situ hybridization; hpf: hours-post-fertilization; NAMP: naphtol-AS-MX-phosphate; POD: horseradish peroxidase; TSA: tyramide signal amplification; WISH: whole-mount in situ hybridization

\section{Acknowledgements}

We thank our colleagues for sharing plasmids for RNA probes. This study was supported by funds from Stockholm County Council and Karolinska Institute. Confocal microscopy was done at the Live Cell Imaging unit, Department of Biosciences and Nutrition, Karolinska Institutet, Huddinge, Sweden, supported by grants from the Knut and Alice Wallenberg Foundation, the Swedish Research Council and the Centre for Biosciences.

\section{Authors' contributions}

$\mathrm{GL}$ and IS did the experiments. GH designed the project, GH wrote the manuscript and GL designed the figures. All three authors contributed to finalizing the manuscript, read and approved the final version of the manuscript.

\section{Competing interests}

The authors declare that they have no competing interests.

Received: 12 April 2011 Accepted: 4 July 2011 Published: 4 July 2011

\section{References}

1. Hafen E, Levine M, Garber RL, Gehring WJ: An improved in situ hybridization method for the detection of cellular RNAs in Drosophila tissue sections and its application for localizing transcripts of the homeotic Antennapedia gene complex. EMBO J 1983, 2:617-23.

2. Tautz D, Pfeifle C: A non-radioactive in situ hybridization method for the localization of specific RNAs in Drosophila embryos reveals translational control of the segmentation gene hunchback. Chromosoma 1989, 98:81-5.

3. Hauptmann G: Two-color detection of mRNA transcript localizations in fish and fly embryos using alkaline phosphatase and beta-galactosidase conjugated antibodies. Dev Genes Evol 1999, 209:317-21.

4. Hauptmann G: One-, two-, and three-color whole-mount in situ hybridization to Drosophila embryos. Methods 2001, 23:359-72. 
5. Hauptmann G, Gerster T: Multicolor whole-mount in situ hybridization Methods Mol Biol 2000, 137:139-48.

6. Jowett T: Double in situ hybridization techniques in zebrafish. Methods 2001, 23:345-58.

7. O'Neill JW, Bier E: Double-label in situ hybridization using biotin and digoxigenin-tagged RNA probes. Biotechniques 1994, 17:870, 874-5.

8. Hauptmann G, Gerster T: Two-color whole-mount in situ hybridization to vertebrate and Drosophila embryos. Trends Genet 1994, 10:266.

9. Jowett $T$, Lettice $L$ : Whole-mount in situ hybridizations on zebrafish embryos using a mixture of digoxigenin- and fluorescein-labelled probes. Trends Genet 1994, 10:73-4

10. Hauptmann G, Gerster T: Regulatory gene expression patterns reveal transverse and longitudinal subdivisions of the embryonic zebrafish forebrain. Mech Dev 2000, 91:105-18.

11. Hauptmann G, Söll I, Gerster T: The early embryonic zebrafish forebrain is subdivided into molecularly distinct transverse and longitudinal domains. Brain Res Bull 2002, 57:371-5.

12. Hauptmann G, Belting HG, Wolke U, Lunde K, Söll I, Abdelilah-Seyfried S, Prince $V$, Driever W: spiel ohne grenzen/pou2 is required for zebrafish hindbrain segmentation. Development 2002, 129:1645-55.

13. Chandrasekar G, Lauter G, Hauptmann G: Distribution of corticotropinreleasing hormone in the developing zebrafish brain. J Comp Neurol 2007, 505:337-51.

14. Bräutigam L, Hillmer JM, Söll I, Hauptmann G: Localized expression of urocortin genes in the developing zebrafish brain. J Comp Neurol 2010, 518:2978-95

15. Murdoch A, Jenkinson EJ, Johnson GD, Owen JJ: Alkaline phosphatase-fast red, a new fluorescent label. Application in double labelling for cell cycle analysis. J Immunol Methods 1990, 132:45-9.

16. Larison KD, BreMiller R, Wells KS, Clements I, Haugland RP: Use of a new fluorogenic phosphatase substrate in immunohistochemical applications. J Histochem Cytochem 1995, 43:77-83.

17. Bueno D, Skinner J, Abud H, Heath JK: Double in situ hybridization on mouse embryos for detection of overlapping regions of gene expression. Trends Genet 1996, 12:385-7.

18. Jowett T, Yan YL: Double fluorescent in situ hybridization to zebrafish embryos. Trends Genet 1996, 12:387-9.

19. Bobrow MN, Harris TD, Shaughnessy KJ, Litt GJ: Catalyzed reporter deposition, a novel method of signal amplification. Application to immunoassays. J Immunol Methods 1989, 125:279-85.

20. Clay H, Ramakrishnan L: Multiplex fluorescent in situ hybridization in zebrafish embryos using tyramide signal amplification. Zebrafish 2005, 2:105-11.

21. Denkers N, Garcia-Villalba P, Rodesch CK, Nielson KR, Mauch TJ: FISHing for chick genes: Triple-label whole-mount fluorescence in situ hybridization detects simultaneous and overlapping gene expression in avian embryos. Dev Dyn 2004, 229:651-7.

22. Kosman D, Mizutani CM, Lemons D, Cox WG, McGinnis W, Bier E: Multiplex detection of RNA expression in Drosophila embryos. Science 2004, 305:846.

23. Tessmar-Raible K, Steinmetz PR, Snyman H, Hassel M, Arendt D: Fluorescent two-color whole mount in situ hybridization in Platynereis dumerilii (Polychaeta, Annelida), an emerging marine molecular model for evolution and development. Biotechniques 2005, 39:460, 462, 464

24. Lecuyer E, Parthasarathy N, Krause HM: Fluorescent in situ hybridization protocols in Drosophila embryos and tissues. Methods Mol Biol 2008 420:289-302.

25. Lauter G, Söll I, Hauptmann G: Multicolor fluorescent in situ hybridization to define abutting and overlapping gene expression in the embryonic zebrafish brain. Neural Dev 2011, 6:10.

26. Hauptmann G, Gerster T: Multicolour whole-mount in situ hybridization to Drosophila embryos. Development Genes and Evolution 1996, 206:292-295

27. van Gijlswijk RP, Wiegant J, Raap AK, Tanke HJ: Improved localization of fluorescent tyramides for fluorescence in situ hybridization using dextran sulfate and polyvinyl alcohol. J Histochem Cytochem 1996 44:389-92.

28. Minton AP: Implications of macromolecular crowding for protein assembly. Curr Opin Struct Biol 2000, 10:34-9.
29. Hauptmann G, Gerster T: Combinatorial expression of zebrafish Brn-1- and Brn-2-related POU genes in the embryonic brain, pronephric primordium, and pharyngeal arches. Dev Dyn 2000, 218:345-58.

30. Bolte S, Cordelieres FP: A guided tour into subcellular colocalization analysis in light microscopy. J Microsc 2006, 224:213-32.

31. Puelles $L$, Rubenstein $J$ : Expression patterns of homeobox and other putative regulatory genes in the embryonic mouse forebrain suggest a neuromeric organization. Trends Neurosci 1993, 16:472-9.

32. Puelles $L$, Rubenstein $J L$ : Forebrain gene expression domains and the evolving prosomeric model. Trends Neurosci 2003, 26:469-76.

33. Wullimann MF, Puelles L: Postembryonic neural proliferation in the zebrafish forebrain and its relationship to prosomeric domains. Anat Embryol (Berl) 1999, 199:329-48.

34. Qiu M, Shimamura K, Sussel L, Chen S, Rubenstein JL: Control of anteroposterior and dorsoventral domains of Nkx-6.1 gene expression relative to other Nkx genes during vertebrate CNS development. Mech Dev 1998, 72:77-88

35. Choi HM, Chang JY, Trinh le A, Padilla JE, Fraser SE, Pierce NA: Programmable in situ amplification for multiplexed imaging of mRNA expression. Nat Biotechnol 2010, 28:1208-12.

36. Kloosterman WP, Wienholds E, de Bruijn E, Kauppinen S, Plasterk RH: In situ detection of miRNAs in animal embryos using LNA-modified oligonucleotide probes. Nat Methods 2006, 3:27-9.

37. Serluca FC, Fishman MC: Pre-pattern in the pronephric kidney field of zebrafish. Development 2001, 128:2233-41.

38. Krauss S, Concordet JP, Ingham PW: A functionally conserved homolog of the Drosophila segment polarity gene hh is expressed in tissues with polarizing activity in zebrafish embryos. Cell 1993, 75:1431-44.

39. Püschel AW, Gruss P, Westerfield M: Sequence and expression pattern of pax-6 are highly conserved between zebrafish and mice. Development 1992, 114:643-51.

40. Cheesman SE, Layden MJ, Von Ohlen T, Doe CQ, Eisen JS: Zebrafish and fly Nkx6 proteins have similar CNS expression patterns and regulate motoneuron formation. Development 2004, 131:5221-32.

41. Long S, Rebagliati M: Sensitive two-color whole-mount in situ hybridizations using digoxygenin- and dinitrophenol-labeled RNA probes. Biotechniques 2002, 32:494-500.

42. Hopman AH, Ramaekers FC, Speel EJ: Rapid synthesis of biotin-, digoxigenin-, trinitrophenyl-, and fluorochrome-labeled tyramides and their application for In situ hybridization using CARD amplification. J Histochem Cytochem 1998, 46:771-7.

doi:10.1186/1471-213X-11-43

Cite this article as: Lauter et al.: Two-color fluorescent in situ hybridization in the embryonic zebrafish brain using differential detection systems. BMC Developmental Biology 2011 11:43.

\section{Submit your next manuscript to BioMed Central and take full advantage of:}

- Convenient online submission

- Thorough peer review

- No space constraints or color figure charges

- Immediate publication on acceptance

- Inclusion in PubMed, CAS, Scopus and Google Scholar

- Research which is freely available for redistribution 